\title{
Predicting Missing Field Boundaries to Increase Per-Field Classification Accuracy
}

Paul Aplin and Peter M. Atkinson

\begin{abstract}
A new technique for predicting missing field boundaries was developed to increase the accuracy of per-field classification. This technique is based on a comparison of within-field modal land-cover proportion and local variance. Analysis was performed on 4-m and 20-m spatial resolution imagery derived from Compact Airborne Spectrographic Imager (CASI) data, to simulate the difference in land-cover classification accuracy between multispectral Ikonos and Satellite Pour l'Observation de la Terre (SPOT) High Resolution Visible (HRV) imagery. Initially, per-pixel classification was performed, followed by perfield classification. The technique for detecting missing boundaries was then implemented, and per-field classification was carried out a second time using updated field boundary data. Finally, an accuracy assessment was performed. The results demonstrate that classification was significantly more accurate when the missing boundary flag was used, and that simulated Ikonos imagery was considerably more accurate for this purpose than simulated SPOT HRV imagery.
\end{abstract}

\section{Introduction}

For many years the accuracy of land-cover mapping from satellite sensors was limited by the relatively coarse spatial resolution of the imagery available (Townshend, 1992). Until the late 1990s, the finest spatial resolution imagery used widely for this purpose was that of the Satellite Pour l'Observation de la Terre (SPOT) High Resolution Visible (HRV) sensor. Multispectral SPOT HRV imagery has a spatial resolution of $20 \mathrm{~m}$. While this is adequate for accurate regional-scale landcover mapping, local detail may be obscured (Fuller et al., 1994). Since September 1999, 4-m spatial resolution multispectral imagery has been available from the Ikonos satellite sensor. Subsequently, 3.2-m spatial resolution multispectral imagery became available from the QuickBird satellite sensor, launched in October 2001 (DigitalGlobe, URL, http://www. digitalglobe.com, last accessed 07 September 2003), and another spatial resolution satellite sensor, OrbView-3, is due for launch in 2003 (ORBIMAGE, personal communication, 2002). Like Ikonos, OrbView-3 will generate multispectral imagery with a spatial resolution of $4 \mathrm{~m}$. This relatively fine spatial resolution has the potential to provide accurate land-cover mapping at the local scale (Aplin et al., 1997).

Traditionally, land-cover mapping from remotely sensed imagery has been performed by per-pixel classification, whereby each image pixel is associated with one or more land-cover classes (Mather, 1999). One problem associated with the use of fine spatial resolution imagery for per-pixel

P. Aplin is with the School of Geography, The University of Nottingham, University Park, Nottingham NG7 2RD, United Kingdom (paul.aplin@nottingham.ac.uk).

P. Atkinson is with the Department of Geography, University of Southampton, Highfield, Southampton SO17 1BJ, United Kingdom (P.M.Atkinson@soton.ac.uk). land-cover classification is over-sampling, whereby the imagery is too detailed to differentiate accurately between features of interest, instead resolving within-feature variation (Irons et al., 1985; Cushnie, 1987; Smith et al., 2002). For instance, where the feature of interest is a woodland area, fine spatial resolution imagery may resolve individual trees and patches of grass separating trees, leading to inaccurate perpixel classification of woodland. Specifically, woodland pixels may be classified incorrectly as grass. The problem, viewed more generally, is that the spatial resolution is too fine relative to the level of spatial generalization desired, or specified, by the user. To solve this, per-parcel (or, per-field) classification may be performed whereby each field, rather than each pixel, is assigned membership to a land-cover class or classes. This removes within-field variation, thereby potentially increasing classification accuracy (Ortiz et al., 1997; Aplin et al., 1999a; Berberoglu and Curran, 2000).

Commonly, per-field classification is performed by integrating remotely sensed imagery and cartographic vector data. One major problem associated with this approach is that of missing field boundaries. Where field boundaries are missing from (or where the vectors representing them are unclosed in) the cartographic data, entire fields can be classified incorrectly (Aplin et al., 1998). This is a particular problem when classifying large fields of rural land cover. For example, where two fields share a missing boundary, the smaller field may be misclassified in its entirety. To avoid this type of misclassification, it is necessary to identify such fields and locate missing field boundaries.

This paper presents a new technique for predicting missing field boundaries to increase the accuracy of subsequent per-field classification. This technique is based on a comparison of the within-field proportion of modal land cover (the dominant land-cover class) and local variance (a descriptor of spatial variation). Such a comparison provides a simple characterization of the variation in land cover within each field and, therefore, an indication of the likelihood of missing boundaries. Analysis is performed on both 4-m and 20-m spatial resolution imagery, to estimate the difference in land-cover classification accuracy between Ikonos and SPOT HRV imagery. Initially, the methods used in this research are outlined, followed by a description of the study area and data. Then, the six-stage analytical procedure is presented, followed by some topics of discussion and, finally, five concluding points.

\section{Methods}

Four methods are described: (1) per-pixel classification, (2) per-field classification, (3) missing boundary flag, and (4) accuracy assessment.

Photogrammetric Engineering \& Remote Sensing Vol. 70, No. 1, January 2004, pp. 141-149.

0099-1112/04/7001-0141/\$3.00/0 (C) 2004 American Society for Photogrammetry and Remote Sensing 


\section{Per-Pixel Classification}

One widely used method of per-pixel classification is the maximum-likelihood (ML) algorithm, whereby pixels are assigned to the class to which they are most likely to belong. This assignment is based on their relationship with, for each class, the mean and variance-covariance matrix characterizing the distributions of the training data in feature space (Settle and Briggs, 1987; Thomas et al., 1987; Tso and Mather, 2001).

The Mahalanobis distance $M_{k j}$ (for class $k$ at pixel location $j$ ) takes into account the variance-covariance matrix $\mathbf{V}_{k}$ associated with a given class $k$ : i.e.,

$$
M_{k j}=\left(\mathbf{z}\left(\mathbf{x}_{j}\right)-\mathbf{u}_{k}\right)^{\mathrm{T}} \mathbf{V}_{k}^{-1}\left(\mathbf{z}\left(\mathbf{x}_{j}\right)-\mathbf{u}_{k}\right)
$$

where $\mathbf{z}\left(\mathbf{x}_{j}\right)$ is a vector of pixel values in $\{i=1, \ldots, n\}$ wavebands at pixel location $\mathbf{x}_{j}$ and $\mathbf{u}_{k}$ is the vector of means in $n$ wavebands for class $k$. Using the Mahalanobis distance, it is possible to determine a predicted (Gaussian) probability density for each class of interest: i.e.,

$$
p\left(z\left(\mathbf{x}_{j}\right) \mid k\right)=\frac{1}{(2 \pi)^{n / 2}\left|\mathbf{V}_{k}\right|^{1 / 2}} \exp \left[-1 / 2 M_{k j}\right]
$$

where $p\left(\mathbf{z}\left(\mathbf{x}_{j}\right) \mid k\right)$ is the probability density for pixel value $\mathbf{z}\left(\mathbf{x}_{j}\right)$ as a member of class $k$ (Thomas et al., 1987; Foody et al., 1992).

To use the above probabilities in a maximum-likelihood classification, these must be converted to a posteriori probabilities. The a posteriori probability of obtaining class $k$ given pixel $\mathbf{z}\left(\mathbf{x}_{i}\right), L\left(k \mid \mathbf{z}\left(\mathbf{x}_{j}\right)\right)$ may be obtained from Bayes' Theorem, which may be written fully to include the a priori probabilities: i.e.,

$$
L\left(k \mid z\left(\mathbf{x}_{j}\right)\right)=\frac{P_{k} \cdot p\left(z\left(\mathbf{x}_{j}\right) \mid k\right)}{\sum_{r=1}^{t} P_{r} \cdot p\left(z\left(\mathbf{x}_{j}\right) \mid r\right)}
$$

where $P_{k}$ is the a priori probability of membership in class $k$. ML classification is a relatively simple and well known computational procedure available in many digital image processing systems. Despite certain drawbacks, such as the assumptions that the data are normally distributed and the classes to be predicted are discrete (Foody, 1996), ML classification has been used widely and has performed relatively accurately (Palacio-Prieto and Luna-González, 1996; San MiguelAyanz and Biging, 1997; Cortijo and De La Blanca, 1998; Chan et al., 2001; Frizzelle and Moody, 2001). Although alternative non-parametric classification techniques, such as artificial neural networks (Yang et al., 1999; Ji, 2000), have been shown to yield greater accuracy than the ML classifier in certain circumstances, the ML classifier will perform accurately if the Gaussian model is appropriate.

\section{Per-Field Classification}

There are various ways in which per-field classification can be performed. It is possible to generate a per-field classification using only remotely sensed imagery by applying techniques such as edge detection or region growing to determine the field boundaries (e.g., Smith et al., 1998). Commonly, however, per-field classification is performed by integrating raster remotely sensed imagery with vector cartographic data (Janssen and Molenaar, 1995; Ortiz et al., 1997; Aplin et al., 1999a), although it should be noted that error may be introduced where cartographic boundaries are not well matched with natural boundaries within the imagery. That is, the results of such per-field classification are dependent on the accuracy of the cartographic data.
Per-field classification involving the integration of raster and vector data is often achieved by rasterizing cartographic data for combination with remotely sensed imagery, although it is possible to vectorize the remotely sensed imagery and integrate the image and cartographic data on a vector basis (Mattikali et al., 1995). The remotely sensed imagery and cartographic data may be integrated at one of three stages in the per-field classification procedure: (1) before classification (pre-classifier stratification), (2) during classification (classifier modification), or (3) after classification (post-classifier sorting) (Mason et al., 1988). Most recent examples of perfield classification have employed only the latter two. For example, Westmoreland and Stow (1992), Wang et al. (1997), and Smith et al. (1997) integrate cartographic data with remotely sensed imagery during classification to assess landcover change on a per-field basis. Alternatively, several studies have classified land cover on a per-pixel basis before integrating the classified image with cartographic data for perfield classification. The land-cover class of each field is predicted by a statistic, such as the modal class for all pixels within that field (Janssen et al., 1990; White et al., 1995; Aplin et al., 1999a). A benefit of this final approach is that it may be possible to use certain by-products of per-field classification, such as per-field modal land cover proportions (the proportion of each field occupied by the modal land-cover class) in subsequent analysis.

\section{Missing Boundary Flag}

The problem of missing field boundaries in per-field classification may be reduced by employing statistical analysis to identify fields with missing or incomplete boundaries. The procedure employed in this paper involves comparing the proportional coverage of the modal land-cover class and the local variance of each field. While the modal land-cover proportion can be extracted from per-field classification (as indicated above), the local variance requires further processing.

\section{Local Variance}

Local variance has been used to determine the optimal spatial resolution of remotely sensed imagery (Woodcock and Strahler, 1987; Tsang and Barnsley, 1996). Where spatial resolution is at a higher spatial frequency than land-cover variation (e.g., finer than land-cover features of interest), proximate pixel values will tend to be similar and local variance will be relatively small. As spatial resolution coarsens, proximate pixel values become less similar and local variance increases. Local variance is calculated as the mean value over an image of the standard deviation for a 3- by 3-pixel moving window: i.e.,

$$
\sigma^{2}=\sum \frac{x^{2}}{n-1}-\left(\frac{\sum x}{n(n-1)}\right)^{2} .
$$

That is, for each pixel in the image, where the pixel is central to the moving window, the standard deviation of the nine pixel values in the window is calculated. The mean of these standard deviations throughout the image is most often used (Woodcock and Strahler, 1987; Tsang and Barnsley, 1996).

Given that the approach presented here is a post-classification search for fields likely to have a missing boundary, the local variance of classified pixels per field was predicted. It should be noted that this is a variation on the traditional approach to calculating local variance. The classified per-pixel image and the cartographic vector data were integrated by rasterizing the cartographic data to the same pixel size as the imagery. A 3- by 3-pixel moving window was then passed over the associated data sets and, for each field, the local variance was calculated. Local variance was calculated as the local difference between the central pixel and its neighbors, 
where neighbouring pixels were either the "same class as" or "a different class to" the central pixel. The actual nominal class values were not used in the calculation.

\section{Comparing the Proportion of Modal Land Cover} and Local Variance

Following the extraction of modal land-cover proportions and the prediction of average local variance, the two sets of values were compared. The rationale for use of the two indices is as follows. Where the proportion of modal land cover is small (indicating the presence of multiple land-cover classes) and the local variance is large (indicating a rough texture), fields are likely to be mixed. Where the proportion of modal land cover is large (indicating the presence of a single dominant land-cover class) and the local variance is small (indicating a smooth texture), fields are likely to be homogeneous. Where both the proportion of modal land cover and the local variance are relatively small (indicating a smooth texture but the presence of multiple land-cover classes), fields are likely to comprise homogeneous patches of different land-cover classes, indicating that field boundaries may be missing.

It should be noted that the above procedure does not identify fields with missing boundaries; it identifies fields with a high likelihood of missing boundaries. The benefit of the missing boundary flag, therefore, is to select a subset of fields for manual checking. Examining every field for missing boundaries takes a large amount of time. Examining a subset rather than an entire population of fields saves time.

\section{Automating the Procedure}

To increase ease of use, the procedure for selecting subsets of fields for manual checking can be automated. That is, rather than relying on subjective judgement to select fields, certain criteria can be used to select fields automatically. In this paper, thresholds were set for the proportion of modal land cover and local variance, and only those fields below these thresholds were selected. The position of the thresholds will depend on the nature of the distributions and the requirements of the user. Relatively small thresholds will enable the selection of a small subset for manual checking, but may exclude fields with missing boundaries from the subset. Relatively large thresholds will generate a large subset for manual checking, but should include the majority of fields with missing boundaries in the subset.

\section{Accuracy Assessment}

The final stage of any land-cover classification is accuracy assessment, without which the user does not know the accuracy and, therefore, the utility of the classification (Janssen and Wel, 1994). Generally, accuracy assessment is performed by comparing the classification with reference land-cover data of the study area. One statistical measure used commonly to express classification accuracy is the kappa coefficient of agreement (Congalton, 1991): i.e.,

$$
k=\frac{A_{o}-\sum_{i=1}^{r} X_{i+} X_{+i}}{1-\sum_{i=1}^{r} X_{i+} X_{+i}}
$$

where $A_{o}=$ overall classification accuracy, $r$ is the number of rows, $x_{i+}$ is the marginal total of row $i$, and $x_{+i}$ is the marginal total of column $i$. The kappa coefficient has certain advantages over relatively simple accuracy assessment statistics such as the overall classification accuracy. In particular, the calculation of the kappa coefficient uses all elements of the confusion matrix (rather than simply the main diagonal, as is the case for the overall classification accuracy), and includes a predic-

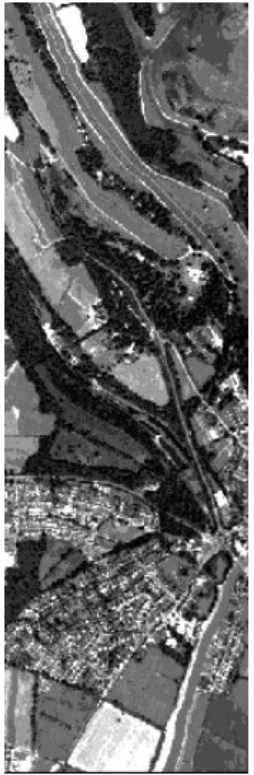

(a)

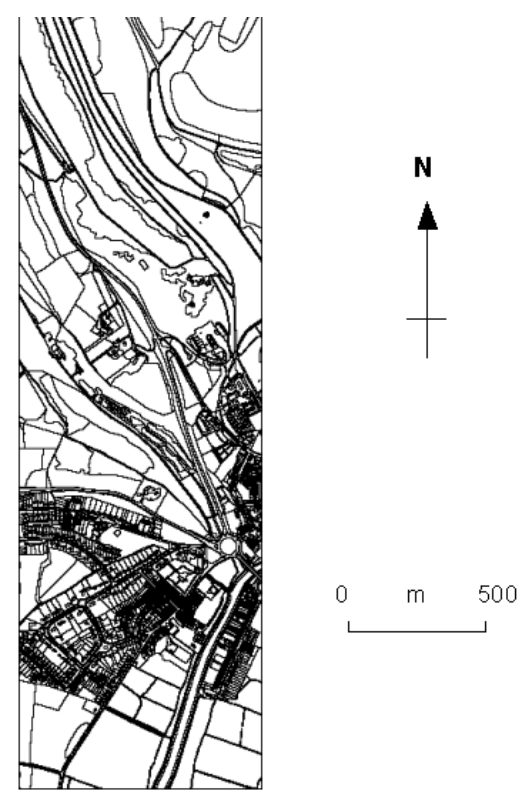

(b)
Figure 1. Arundel study area. (a) Original 4-m spatial resoIution Compact Airborne Spectrographic Imager (CASI) imagery. (b) Land-Line vector data. (Land-Line data reproduced with kind permission of Ordnance Survey (c) Crown Copyright NC/00/1354.)

tion of chance agreement (Stehman, 1997). For a full discussion of accuracy assessment methods, see Nishii and Tanaka (1999).

\section{Study Area and Data}

The study area, covering $0.9 \mathrm{~km}$ by $2.9 \mathrm{~km}$, was located near Arundel, West Sussex, United Kingdom (Figure 1). This area comprised a variety of land-cover types, including semi-natural vegetation in Arundel Country Park to the north, agricultural fields on the western and southern margins, and urban land cover in the village of Arundel in the center.

Compact Airborne Spectrographic Imager (CASI) imagery was acquired on 22 July 1997 and supplied by The National Centre for Environmental Data and Surveillance of the Environment Agency (Figure 1a). The image used for analysis had a spatial resolution of $4 \mathrm{~m}$ and four visible and near-infrared spectral wavebands ( 480 to $520 \mathrm{~nm}, 545$ to $603 \mathrm{~nm}, 660$ to $685 \mathrm{~nm}$, and 845 to $890 \mathrm{~nm}$ ). These specifications were used to approximate those of the Ikonos and OrbView-3 sensors.

Land-Line digital vector data, comprising coded point and line features registered to the British National Grid (BNG), were supplied by the Ordnance Survey (Figure 1b). The vector data were polygonized such that each polygon (field) was identified as a discrete object.

Ground reference data were acquired in early September 1997 through a combination of interviews with residents and a land-cover survey. These two sources of data were combined to generate a comprehensive reference land-cover map of the study area. This map was used for class selection and assessing classification accuracy.

\section{Analysis}

Six stages of analysis were performed: (1) pre-processing, (2) per-pixel classification, (3) per-field classification, (4) missing 
boundary flag, (5) per-field classification following the addition of missing boundaries, and (6) accuracy assessment.

\section{Pre-Processing}

Prior to classification analysis, two pre-processing steps were implemented. First, the CASI image and Land-Line data were coregistered to enable the integrated analysis required for subsequent per-field classification. Because the Land-Line data were already registered to the BNG, the CASI image was registered directly to the Land-Line data. Initially, 35 well distributed ground control points (GCPs) were identified on both data sets (CASI image and Land-Line coverage). The two sets of GCP coordinates were then compared to generate a second-order mathematical transform function. Finally, nearest-neighbor resampling was employed to transform the CASI image to the BNG with a root-mean-square error of $8.64 \mathrm{~m}$.

Second, to enable a comparison between spatial resolutions of $4 \mathrm{~m}$ and $20 \mathrm{~m}$, the original image was degraded to a spatial resolution of $20 \mathrm{~m}$. A relatively simple method of image degradation was employed whereby the spectral values of the pixels in the original CASI image were averaged to derive the spectral values of the pixels in the degraded image. This simple technique of image degradation has been practiced widely (e.g., Heric et al., 1996), although it is acknowledged that some investigators have stressed the need to account for additional factors, such as the signal-to-noise ratio (SNR) and the point spread function (PSF), in the process (e.g., Townshend and Justice, 1988). However, it was believed that the influence of these two factors on the degradation process was relatively minor and the omission of any measures to account for them here did not affect the results significantly. For a fuller discussion on this point, see Aplin et al. (1999b).

\section{Per-Pixel Classification}

Initially, seven land-cover classes were selected for classification (linseed, grassland 1, grassland 2, woodland, bare soil, water, and bright surfaces). The "grassland 1" class comprised relatively short, smooth grasses, while the "grassland 2" class comprised relatively long, rough grasses. A distinction between these two classes may be explained more fully with respect to their land use. The former class was largely agricultural and used for grazing livestock, while the latter was largely untended. A single "woodland" class was used for analysis because wooded areas tended to be characterized by a mixture of deciduous species. Conifers were present in the study area but were relatively few and, where present, tended to combine with dominant deciduous species to form mixed forest stands. Several young woodland plantations were present, planted following severe storms in 1987 which devastated certain areas of woodland throughout the area. The "bright surfaces" class (a combination of asphalt, concrete, rooftiles, and so on) was used to represent urban areas.

Per-pixel classification was performed on the 4-m and 20-m spatial resolution images (Figures $2 \mathrm{a}$ and $2 \mathrm{~b}$, respectively). In each case, the ML classifier was trained on a representative sample of each of the classes, and a supervised classification was performed. In this study, classification was performed to compare per-pixel and per-field techniques rather than to appraise a specific per-pixel classification algorithm. Because it is a wellunderstood technique, ML classification was an appropriate means of achieving this.

\section{Per-Field Classification}

To classify per field, the 4-m and 20 -m spatial resolution perpixel classified images were each combined with the LandLine polygon coverage (Figures 2c and 2d, respectively). Initially, the raster CASI image and vector Land-Line coverage were integrated using a GIS. To achieve this, the Land-Line data were rasterized such that each Land-Line polygon was represented by a pixel or a contiguous group of pixels. The two raster data sets (CASI and Land-Line) were then combined in a single raster grid, enabling joint analysis.

To perform per-field classification, the modal land-cover class (the spatially dominant land-cover class) was assigned to each field (Land-Line polygon) in the combined raster grid. Specifically, the proportion of each field covered by each land-cover class was calculated. Then, for each field the class with the largest number of pixels (the modal land-cover class) was identified. Finally, this class value was reassigned to the entire field in the original vector (non-rasterized) Land-Line coverage.

\section{Missing Boundary Flag}

A major problem was encountered when attempting to classify rural areas on a per-field basis. Numerous field boundaries were missing, which resulted in the misclassification of entire fields. The absence of field boundaries led to the amalgamation of multiple adjoining fields, regardless of any variation in land cover, and resulted in the class of the spatially dominant field (that determining the modal land cover) being assigned incorrectly to the smaller fields. Incomplete or unclosed field boundaries (dangling vector lines) had the same result.

Initially, because the problem of missing field boundaries was associated primarily with large rural fields, a subset of the largest fields within the field site was selected for analysis. Fields with an area of greater than $8000 \mathrm{~m}^{2}$ were selected. This threshold was chosen so that the resulting subset provided an adequate and reasonable sample size and included all major rural fields. Fifty-three fields matched the criterion. Then, for each field, the proportion of modal land cover was extracted from the per-field classification, the local variance was calculated, and the two values were compared.

\section{Comparing Modal Land-Cover Proportion and Local Variance}

The proportion of modal land cover was plotted against the local variance on a per-field basis. This was performed for both 4-m and 20-m spatial resolution CASI imagery (Figures 3a and $3 \mathrm{~b}$, respectively). In general, the local variance of fields was considerably larger at the coarser (20-m) spatial resolution. The mean local variance of the 53 fields at a spatial resolution of $20 \mathrm{~m}$ was greater than 0.28 , while that of the same fields at a spatial resolution of $4 \mathrm{~m}$ was less than 0.20 . This was largely a consequence of the direct relationship between (coarsening) spatial resolution and (decreasing) variance between pixels (Woodcock and Strahler, 1987).

The values representing the proportion of modal land cover for each field were relatively similar between the 4-m and $20-\mathrm{m}$ spatial resolution imagery. The mean proportion of modal land cover for the 53 fields at the finer spatial resolution was slightly less than 67 percent, approximately 3 percent greater than that of the same fields at the coarser spatial resolution. Thus, although the scale of sampling changed, the proportions of land-cover classes remained relatively constant.

\section{Automating the Procedure}

Thresholds were selected to automate the procedure for selecting subsets of fields for manual checking. For each distribution, thresholds were positioned at two thirds of the data range (Figures 3a and $3 \mathrm{~b}$ ), calculated as

$$
\text { threshold }=\min +(2(\max -\min )) / 3 \text {. }
$$

These thresholds are essentially arbitrary, but were deemed appropriate because they enabled the selection of relatively small subsets of the population of fields for manual checking. Subsets of 20 and 25 fields were selected, respectively, using 4-m and 20-m spatial resolution imagery. 


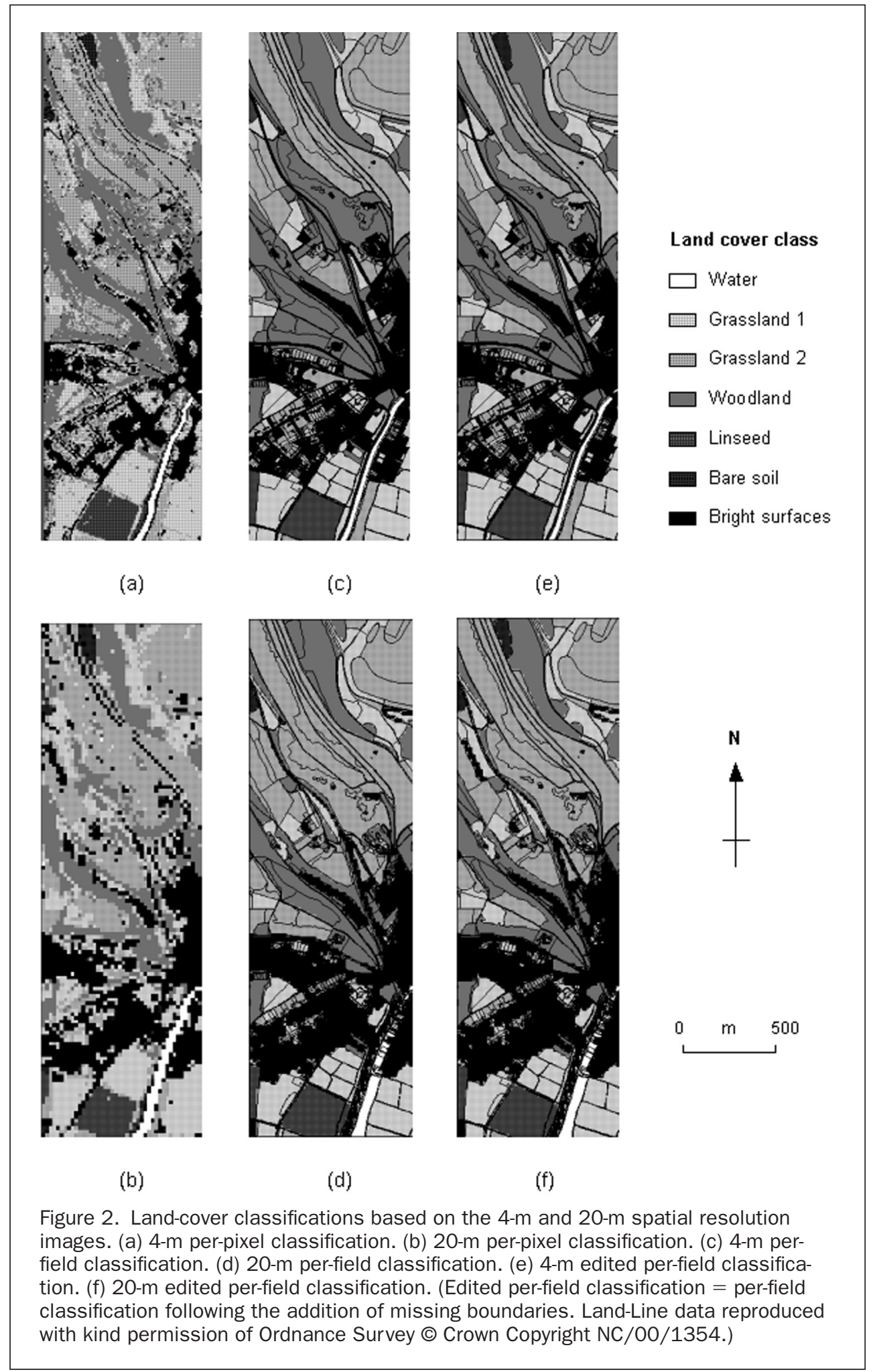

The thresholds segmented the distribution of fields into quadrants, where the quadrants may be used to categorize the likely characteristics of the fields. The upper-left quadrants, where the local variance was large and the proportion of modal land cover was small, identified fields likely to be mixed. The lower-right quadrants, where the local variance was small and the proportion of modal land cover was large, identified fields likely to be homogeneous. For example, the cluster of fields in the lower-right quadrants of the distributions in Figures 3a and 3b were predominantly large, homogeneous agricultural fields. The lower-left quadrants, where both local variance and proportion of modal land cover were small, identified fields likely to have missing boundaries. For example, many of the fields at the lower left of the distributions in Figures 3a and 3b were large agricultural or semi-natural fields with missing boundaries. Fields within the latter quadrant 


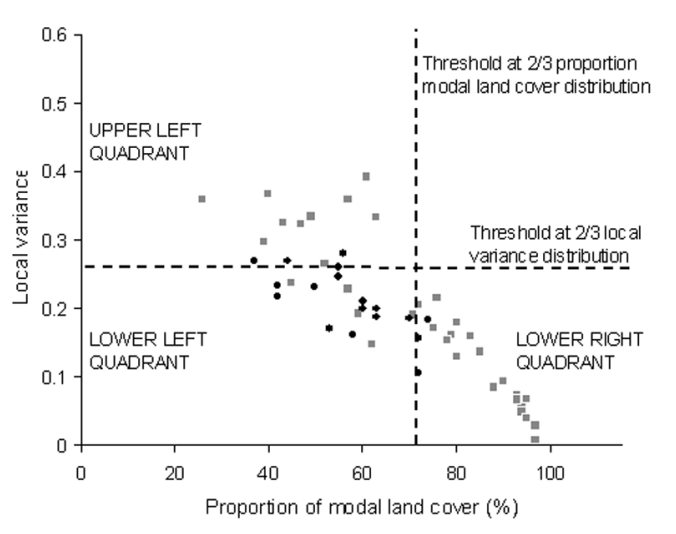

(a)

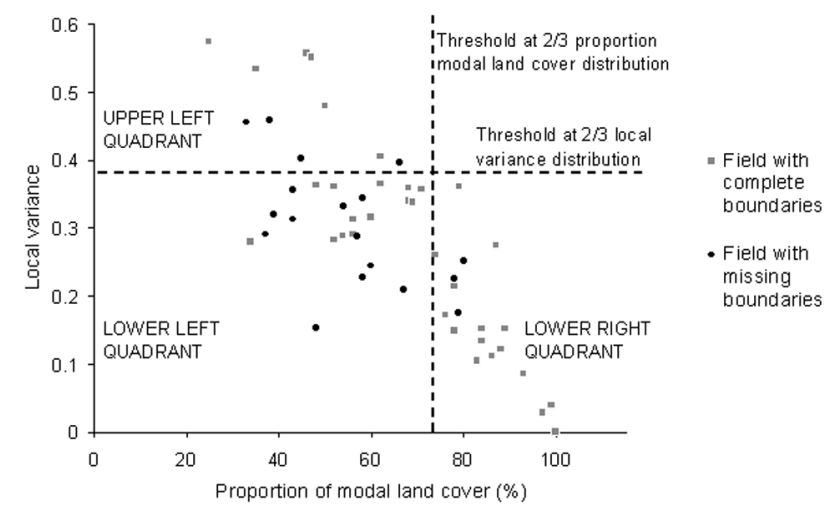

(b)

Figure 3. Relationship between local variance and proportion of modal land cover for (a) 4-m spatial resolution imagery and (b) 20-m spatial resolution imagery.

were subjected to manual checking. Within the 20-field subset selected using 4-m spatial resolution imagery, 14 fields with missing boundaries were identified. Within the 25 -field subset selected using 20-m spatial resolution imagery, 11 fields with missing boundaries were identified.

Following manual checking, the vector data were edited manually to add missing field boundaries, thereby creating more accurate Land-Line polygon coverages (to be used in a second attempt at per-field classification). It should be noted that, for each spatial resolution, only those missing field boundaries identified previously using that spatial resolution were added for subsequent classification. That is, classification of the 4-m spatial resolution imagery used vector data with the addition of missing boundaries for the 14 fields identified using the missing boundary flag. Likewise, 11 fields were edited prior to subsequent classification of the 20-m spatial resolution imagery.
Accuracy of the Missing Boundary Flag

Prior to the continuation of classification analysis, it was deemed useful to assess the accuracy of the missing boundary flag at different spatial resolutions. (It is important to note that this is distinct from the assessment of "classification" accuracy, which follows in the Accuracy Assessment section.) This involved, first, checking manually the entire population of 53 fields used in the missing boundary flag procedure to identify all missing boundaries. Eighteen fields had one or more missing boundaries. This information was then cross-referenced with the plots comparing local variance and proportion of modal land cover (Figures 3a and 3b).

The missing boundary flag was considerably more accurate when using 4-m spatial resolution imagery than 20-m spatial resolution imagery (Table 1). A subset of 20 fields was selected using the former, of which 14 had missing boundaries. Of the 33 fields excluded from selection, 29 had complete boundaries and were, therefore, omitted correctly. In contrast, a larger subset of 25 fields was selected using 20-m spatial resolution imagery. Not only did this subset require more processing time due to the larger number of fields, but only 11 fields with missing boundaries were included. Of those fields omitted, 21 out of 28 had complete boundaries and were omitted correctly. Overall, the missing boundary flag was over 20 percent more accurate when using the finer spatial resolution imagery. This can be illustrated with reference to the distributions of local variance and proportion of modal land cover. Fields with missing boundaries are most densely clustered in the lower-left quadrant of the plot at the finer spatial resolution (Figure 3a) than at the coarser spatial resolution (Figure 3b).

The main reason for the greater accuracy of this procedure at the finer spatial resolution was the greater detail provided by this source of imagery. Considerable spatial autocorrelation was present in the 4-m spatial resolution imagery, enabling a relatively accurate delineation of homogeneous patches of land cover within fields. At the coarser spatial resolution, a considerably greater degree of mixing reduced the accuracy with which such homogeneous patches of land were identified.

Per-Field Classification Following the Addition of Missing Boundaries The per-pixel classified images were re-classified per field using the repaired Land-Line coverages (described in the Automating the Procedure section) for both the 4-m and 20-m spatial resolution CASI images (Figures 2e and 2f, respectively).

\section{Accuracy Assessment}

The accuracies of the six classifications ( $4 \mathrm{~m}$ per-pixel, $4 \mathrm{~m}$ per-field, and $4 \mathrm{~m}$ per-field following the addition of missing boundaries; $20 \mathrm{~m}$ per-pixel, $20 \mathrm{~m}$ per-field, and $20 \mathrm{~m}$ per-field following the addition of missing boundaries) were assessed by comparing the classified images to the reference land-cover map. The results are presented as kappa coefficients (Table 2). A per-pixel accuracy assessment procedure was carried out on both the per-pixel and per-field classifications; for this purpose, the latter were rasterized to the pixel size of the former. Then, random samples of pixels were selected for comparison with the reference land-cover map. It is acknowledged that

TABle 1. ACCURACy OF the Missing Boundary FlaG

\begin{tabular}{lcccccccc}
\hline \multirow{2}{*}{$\begin{array}{l}\text { Spatial } \\
\begin{array}{l}\text { Resolution } \\
\text { of Image }\end{array}\end{array}$} & $\begin{array}{c}\text { Total } \\
\text { Number }\end{array}$ & $\begin{array}{c}\text { Missing } \\
\text { Boundaries }\end{array}$ & $\begin{array}{c}\text { Accuracy } \\
(\%)\end{array}$ & & $\begin{array}{c}\text { Total } \\
\text { Number }\end{array}$ & $\begin{array}{c}\text { Complete } \\
\text { Boundaries }\end{array}$ & $\begin{array}{c}\text { Accuracy } \\
(\%)\end{array}$ & $\begin{array}{c}\text { Accuracy } \\
(\%)\end{array}$ \\
\hline $4 \mathrm{~m}$ & 20 & 14 & 70 & & 33 & 29 & 87.88 & 81.13 \\
$20 \mathrm{~m}$ & 25 & 11 & 44 & & 28 & 21 & 75 & 60.38 \\
\hline
\end{tabular}


Table 2. Kappa Coefficients for Classification Accuracy Assessment

\begin{tabular}{|c|c|c|c|c|c|c|}
\hline \multirow[b]{3}{*}{$\begin{array}{l}\text { Land-Cover } \\
\text { Class }\end{array}$} & \multicolumn{6}{|c|}{ Kappa Coefficients } \\
\hline & \multicolumn{3}{|c|}{ 4-m Spatial Resolution Image } & \multicolumn{3}{|c|}{ 20-m Spatial Resolution Image } \\
\hline & $\begin{array}{c}\text { Per-Pixel } \\
\text { Classification }\end{array}$ & $\begin{array}{c}\text { Original } \\
\text { Per-Field } \\
\text { Classification }\end{array}$ & $\begin{array}{c}\text { Edited } \\
\text { Per-Field } \\
\text { Classification }\end{array}$ & $\begin{array}{c}\text { Per-Pixel } \\
\text { Classification }\end{array}$ & $\begin{array}{c}\text { Original } \\
\text { Per-Field } \\
\text { Classification }\end{array}$ & $\begin{array}{c}\text { Edited } \\
\text { Per-Field } \\
\text { Classification }\end{array}$ \\
\hline Linseed & 0.75 & 0.93 & 0.89 & 0.79 & 0.88 & 0.88 \\
\hline Grassland 1 & 0.56 & 0.68 & 0.73 & 0.58 & 0.79 & 0.81 \\
\hline Grassland 2 & 0.62 & 0.63 & 0.79 & 0.59 & 0.36 & 0.65 \\
\hline Woodland & 0.72 & 0.69 & 0.91 & 0.67 & 0.90 & 0.81 \\
\hline Bare soil & 0.67 & 0 & 1 & 0.38 & 0 & 0.58 \\
\hline Water & 0.82 & 0.79 & 0.75 & 0.28 & 0.06 & 0.05 \\
\hline Bright surfaces & 0.64 & 0.74 & 0.81 & 0.59 & 0.67 & 0.65 \\
\hline Overall & 0.68 & 0.72 & 0.84 & 0.55 & 0.55 & 0.62 \\
\hline
\end{tabular}

(Edited per-field classification $=$ per-field classification following the addition of missing boundaries.)

there are problems associated with assessing the accuracy of per-field classifications on a per-pixel basis. However, this procedure had the benefits of weighting fields according to size (e.g., larger fields were more likely to be selected than smaller fields) and enabling a direct comparison between per-pixel and per-field classifications (Johnsson, 1994; White et al., 1995). A full discussion of the implications of employing different accuracy assessment procedures is provided in Aplin et al. (1999b).

\section{Per-Pixel Versus Per-Field Classification}

Generally, per-pixel classification was slightly less accurate than per-field classification (prior to the addition of missing boundaries). For example, per-pixel classification of the 4-m spatial resolution CASI image generated an overall kappa coefficient of 0.68 , while per-field classification of the same image was marginally more accurate, with an overall kappa coefficient of 0.72 . The main reason for this was spurious misclassification at the per-pixel stage arising because of withinfield spectral variation. Per-field classification, by aggregating to the modal land-cover class, removed such misclassification. However, although per-field classification was more accurate than per-pixel classification for most classes, certain classes were classified with a similar or lower accuracy by the perfield approach. One reason for this was that any increase in accuracy at the per-field stage arising through the removal of spurious within-field classification was offset by a decrease in accuracy caused by missing field boundaries. One extreme example of this was the bare soil class where per-pixel classification generated a kappa coefficient of 0.67 and per-field classification generated a kappa coefficient of 0 . The reason was that only a single field of bare soil was present in the study area and this field was missing from the Land-Line polygon coverage. Consequently, the field was amalgamated with a larger field of woodland and misclassified by the per-field classifier.

\section{Missing Versus Complete Boundaries}

While the original per-field classification of the 4-m spatial resolution CASI image generated an overall kappa coefficient of 0.72 , per-field classification following the addition of missing boundaries generated an overall kappa coefficient of 0.84 . While the accuracy of several classes remained relatively constant between these two, the accuracy of four predominantly rural classes (grassland 1, grassland 2, woodland, bare soil) increased considerably at the latter stage. The reason for this was the inclusion of formerly missing field boundaries in the classification process. This prevented multiple fields from being amalgamated, thereby protecting subsidiary fields from misclassification. For instance, because the field of bare soil was present in the vector data, it was identified correctly. In fact, because this was the only major field of this class, all 50 sample points for bare soil were selected from it and the kappa coefficient was 1.

\section{4-m Versus 20-m Spatial Resolution}

Generally, classification using 4-m spatial resolution CASI imagery was markedly more accurate than classification using 20-m spatial resolution imagery at each of the three stages of analysis (per-pixel, per-field, per-field following the addition of missing boundaries). This was primarily because considerably fewer mixed pixels were present in the finer spatial resolution imagery than in the coarser spatial resolution imagery. For instance, because the bright surfaces class comprised relatively small urban features, the 4-m spatial resolution data enabled more accurate identification of this class than did the 20-m spatial resolution data.

An additional factor affecting only the final stage of classification (per-field following the addition of missing boundaries) was that the missing boundary flag identified more fields with missing boundaries when using the 4-m spatial resolution imagery than when using the 20 -m spatial resolution imagery. This enabled a greater proportion of missing field boundaries to be added prior to per-field classification of the 4-m spatial resolution imagery. Consequently, a greater degree of misclassification occurred in the $20-\mathrm{m}$ spatial resolution imagery through the amalgamation of multiple fields where fields boundaries remained missing.

The most accurate classification overall was the per-field classification following the addition of missing boundaries using 4-m spatial resolution imagery. This generated an overall kappa coefficient of 0.84 . In comparison, the equivalent classification using 20-m spatial resolution imagery generated a kappa coefficient of 0.62 .

\section{Discussion}

The national mapping agency of the United Kingdom, the Ordnance Survey, has recently converted their key spatial data product, Land-Line, into MasterMap ${ }^{\mathrm{TM}}$ as part of the Digital National Framework (DNF). This is a polygon data structure with associated topographical identifiers (TOIDs), and it will form a topographic base to which various attribute data layers will be attached. The role for remote sensing in producing national land-cover and land-use databases should be obvious, 
presenting synergistic opportunities both to the Ordnance Survey and to the remote sensing communities.

The benefits of per-field classification over per-pixel classification have been demonstrated elsewhere (e.g., Aplin et al., 1999a). However, if per-field classification is to be accurate, then it will be necessary to check and potentially update the vector (or polygon) data on which it depends, prior to classification. This paper has demonstrated a simple method for detecting missing field boundaries based on a combination of the modal land cover and the average local variance per field. Three important discussion points follow.

First, "hard" per-pixel classification was used in this research. However, several studies have now demonstrated the benefits of soft classification over hard classification (e.g., Foody, 1999; Melgani et al., 2000; Cheng, 2002; Oki et al., 2002). There is no reason why soft classification should not be applied in the present context. The mode could be replaced by the maximum sum of soft land-cover proportions, and the local variance could be predicted using greater information content. An example of per-field classification using soft classified imagery is provided by Aplin and Atkinson (2001).

Second, the mean local variance was predicted, which implies the decision to use a stationary model of local variance. Such a decision means that potentially useful information is lost. For example, instead of predicting the mean, it may be possible to examine the entire cumulative distribution function (cdf) of values within each field or parcel. Interestingly, using the cdf does not make full use of the available data: the locations of each pixel, which are known, are ignored. One consequence is that it is not possible to say anything about where within each parcel the missing boundaries ought to be located, only that a boundary might be missing. To locate potential boundaries, it is necessary to use an edge detection algorithm, or equivalent, per field. There are many to choose from including the Hough transform and the Sobel filter (Mather, 1999). It may even be possible to use the local variance (mapped within each field) for this purpose. A particularly novel technique that may have application here is the so-called super-resolution mapping approach that takes a soft classification as input (described above) and maps landcover boundaries within pixels (Tatem et al., 2001; Tatem et al., 2002). It is clear that the mean local variance represents only one relatively simple approach from the range of possibilities (e.g., Verhoeye and De Wulf, 2002).

Third, in this paper, arbitrary thresholds were used to predict missing boundaries from the proportion of modal land cover and the local variance. Many alternative approaches exist. For example, supervised classification could be used to potentially increase the accuracy of prediction. For example, for a large area containing many polygons, a small subset of polygons with (and without) missing boundaries could be used to train a supervised classifier. We chose to use untrained thresholds because (1) they corresponded closely to the rationale given in the comparing Modal Land-Cover Proportion and Local Variance section for use of the proportion of modal land cover and local variance (that is, they illustrate the concept well), and (2) such a procedure might be useful where training is not viable (e.g., very large areas of interest). In any case, the purpose of this paper was to explore and illustrate the potential use of the two variables, not to design the technique used to extract information from them.

\section{Conclusions}

Five specific conclusions can be made:

- per-pixel classification of land cover resulted in misclassification due to within-field variation;

- per-field classification removed this source of misclassification but missing field boundaries led to entire fields being misclassified;
- missing boundaries were predicted by comparing within-field modal land-cover proportion and local variance, increasing per-field classification accuracy subsequently;

- 4-m spatial resolution imagery was considerably more accurate than 20-m spatial resolution imagery for predicting missing boundaries fields; and, therefore,

- 4-m spatial resolution imagery was considerably more accurate than $20-\mathrm{m}$ spatial resolution imagery for classifying land cover in rural areas.

\section{References}

Aplin, P., P.M. Atkinson, and P.J. Curran, 1997. Fine spatial resolution satellite sensors for the next decade, International Journal of Remote Sensing, 18:3873-3881.

1998. Identifying missing field boundaries to increase the accuracy of per-field classification of fine spatial resolution satellite sensor imagery, Proceedings of the 27th International Symposium on Remote Sensing of Environment: Information for Sustainability, 08-12 June, Tromso, Norway, Norwegian Space Centre, Oslo, Norway, pp. 399-402.

, 1999a. Fine spatial resolution simulated satellite sensor imagery for land cover mapping in the UK, Remote Sensing of Environment, 68:206-216.

1999b. Per-field classification of land use using the forthcoming very fine spatial resolution satellite sensors: problems and potential solutions, Advances in Remote Sensing and GIS Analysis (P. Atkinson and N. Tate, editors), John Wiley, Chichester, United Kingdom, pp. 219-239.

Aplin, P., and P.M. Atkinson, 2001. Sub-pixel land cover mapping for per-field classification, International Journal of Remote Sensing, 22:2853-2858.

Berberoglu, S., and P.J. Curran, 2000. Utilising texture measures in an artificial neural network for the per-field classification of agricultural land cover in the Mediterranean, Aspects of Applied Biology, $60: 21-28$.

Chan, J.C.-W., K.-P. Chan, and A.G.-O. Yeh, 2001. Detecting the nature of change in an urban environment: A comparison of machine learning algorithms, Photogrammetric Engineering \& Remote Sensing, 67:213-225.

Cheng, T., 2002. Fuzzy objects: their changes and uncertainties, Photogrammetric Engineering \& Remote Sensing, 68:41-49.

Congalton, R.G., 1991. A review of assessing the accuracy of classifications of remotely sensed data, Remote Sensing of Environment, $37: 35-46$.

Cortijo, F.J., and N.P. De La Blanca, 1998. Improving classical contextual classifications, International Journal of Remote Sensing, 19:1591-1613.

Cushnie, J.L., 1987. The interactive effect of spatial resolution and degree of internal variability within land-cover types on classification accuracies, International Journal of Remote Sensing, 8:15-29.

Foody, G.M., 1996. Approaches for the production and evaluation of fuzzy land cover classifications from remotely-sensed data, International Journal of Remote Sensing, 17:1317-1340.

, 1999. The continuation of classification fuzziness in thematic mapping, Photogrammetric Engineering \& Remote Sensing, 65: 443-451.

Foody, G.M., N.A. Campbell, N.M. Trodd, and T.F. Wood, 1992. Derivation and applications of probabilistic measures of class membership from the maximum-likelihood classification, Photogrammetric Engineering \& Remote Sensing, 58:1335-1341.

Frizzelle, B.G., and A. Moody, 2001. Mapping continuous distributions of land cover: A comparison of maximum-likelihood estimation and artificial neural networks, Photogrammetric Engineering \& Remote Sensing, 67:693-705.

Fuller, R.M., G.B. Groom, and A.R. Jones, 1994. The land cover map of Great Britain: An automated classification of Landsat Thematic Mapper data, Photogrammetric Engineering \& Remote Sensing, 60:553-562.

Heric, M., C. Lucas, and C. Devine, 1996. The Open Skies Treaty: Qualitative utility evaluations of aircraft reconnaissance and 
commercial satellite imagery, Photogrammetric Engineering \& Remote Sensing, 62:279-284.

Irons, J.R., B.L. Markham, R.F. Nelson, D.L. Toll, D.L. Williams, R.S. Latty, and M.L. Stauffer, 1985. The effects of spatial resolution on the classification of Thematic Mapper data, International Journal of Remote Sensing, 6:1385-1403.

Janssen, L.L.F., M.N. Jaarsma, and T.M. Van der Linden, 1990. Integrating topographic data with remote sensing for land-cover classification, Photogrammetric Engineering \& Remote Sensing, 56: 1503-1506.

Janssen, L.L.F., and F.J.M. van der Wel, 1994. Accuracy assessment of satellite derived land-cover data: A review, Photogrammetric Engineering \& Remote Sensing, 60:419-426.

Janssen, L.L.F., and M. Molenaar, 1995. Terrain objects, their dynamics and their monitoring by the integration of GIS and remote sensing, IEEE Transactions on Geoscience and Remote Sensing, 33:749-758.

Ji, C.Y., 2000. Land-use classification of remotely sensed data using Kohonen self-organizing feature map neural networks, Photogrammetric Engineering \& Remote Sensing, 66:1451-1460.

Johnsson, K., 1994. Segment-based land-use classification from SPOT satellite data, Photogrammetric Engineering \& Remote Sensing, 60:47-53.

Mason, D.C., D.G. Corr, A. Cross, D.C. Hogg, D.H. Lawrence, M. Petrou, and A.M. Tailor, 1988. The use of digital map data in the segmentation and classification of remotely-sensed images, International Journal of Geographical Information Systems, 9:195-215.

Mather, P.M., 1999. Computer Processing of Remotely-Sensed Images: An Introduction, Second Edition, John Wiley, Chichester, United Kingdom, 292 p.

Mattikalli, N.M., B.J. Devereux, and K.S. Richards, 1995. Integration of remotely sensed satellite images with a geographical information system, Computers and Geosciences, 21:947-956.

Melgani, F., B.A. Al Hashemy, and S.M. Taha, 2000. An explicit fuzzy supervised classification method for multispectral remote sensing images, IEEE Transactions on Geoscience and Remote Sensing, $38: 287-295$

Nishii, R., and S. Tanaka, 1999. Accuracy and inaccuracy assessments in land-cover classification, IEEE Transactions on Geoscience and Remote Sensing, 37:491-498.

Oki, K., H. Oguma, and M. Sugita, 2002. Subpixel classification of alder trees using multitemporal Landsat Thematic Mapper imagery, Photogrammetric Engineering \& Remote Sensing, 68:77-82.

Ortiz, M.J., A.R. Formaggio, and J.C.N. Epiphanio, 1997. Classification of croplands through integration of remote sensing, GIS and historical database, International Journal of Remote Sensing, 18: 95-105.

Palacio-Prieto, J.L., and L. Luna-González, 1996. Improving spectral results in a GIS context, International Journal of Remote Sensing, 17:2201-2209.

San Miguel-Ayanz, J., and G.S. Biging, 1997. Comparison of singlestage and multi-stage classification approaches for cover type mapping with TM and SPOT data, Remote Sensing of Environment, 59:92-104.

Settle, J.J., and S.A. Briggs, 1987. Fast maximum likelihood classification of remotely-sensed imagery, International Journal of Remote Sensing, 8:723-734.

Smith, G., R. Fuller, G. Amable, C. Costa, and B. Devereux, 1997. CLassification of Environment with VEctor-and Raster Mapping (CLEVER mapping), Proceedings of GISRUK97, 09-11 April, University of Leeds, Leeds, United Kingdom, pp. 70-72.
Smith, G.M., R.M. Fuller, and B.J. Devereux, 1998. An integrated vector/raster system for land cover mapping, IEE Coloquium Digest-Integrated Systems for Commercial Remote Sensing Applications, Institute of Electrical Engineers, London, United Kingdom, pp. 2/1-2/6.

Smith, J.H., J.D. Wickham, S.V. Stehman, and L. Yang, 2002. Impacts of patch size and land-cover heterogeneity on thematic image classification accuracy, Photogrammetric Engineering \& Remote Sensing, 68:65-70.

Stehman, S.V., 1997. Selecting and interpreting measures of thematic classification accuracy, Remote Sensing of Environment, 62: 77-89.

Tatem, A.J., H.G. Lewis, P.M. Atkinson, and M.S. Nixon, 2001. Land cover mapping from remotely sensed images at the sub-pixel scale using a Hopfield neural network, IEEE Transactions on Geoscience and Remote Sensing, 39:781-796.

2002. Super-resolution land cover pattern prediction using a Hopfield Neural Network, Remote Sensing of Environment, 79: $1-14$.

Thomas, I.L., V.M. Benning, and N.P. Ching, 1987. Classification of Remotely Sensed Images, Adam Hilger, Bristol, United Kingdom, $256 \mathrm{p}$.

Townshend, J.R.G., 1992. Land cover, International Journal of Remote Sensing, 13:1319-1328.

Townshend, J.R.G., and C.O. Justice, 1988. Selecting the spatial resolution of satellite sensors required for global monitoring of land transformations, International Journal of Remote Sensing, 9:187-236

Tsang, T., and M. Barnsley, 1996. A comparative analysis of various methods for quantifying landscape heterogeneity, Proceedings of RSS'96: Remote Sensing Science and Industry, 11-14 September, University of Durham, Durham, United Kingdom (Remote Sensing Society, Nottingham, United Kingdom), pp. 513-520.

Tso, B.C.K., and P.M. Mather, 2001. Classification Methods for Remotely Sensed Data, Taylor and Francis, New York, N.Y., 332 p.

Verhoeye, K.J., and R. De Wulf, 2002. Land cover mapping at subpixel scales using linear optimization techniques, Remote Sensing of Environment, 79:96-104.

Wang, R.S.M., S.A. Roberts, and N.D. Efford, 1997. An object-based approach to integrate Landsat TM data within a GIS context for detecting land use changes at urban-rural fringe areas, Proceedings of RSS'97: Observations and Interactions, 02-04 September, University of Reading, Reading, United Kingdom (Remote Sensing Society, Nottingham, United Kingdom), pp. 179-184.

Westmoreland, S., and D.A. Stow, 1992. Category identification of changed land-use polygons in an integrated image processing/ geographic information system, Photogrammetric Engineering \& Remote Sensing, 58:1593-1599.

White, J.D., G.C. Kroh, and J.E. Pinder III, 1995. Forest mapping at Lassen Volcanic National Park, California, using Landsat TM data and a geographical information system, Photogrammetric Engineering \& Remote Sensing, 61:299-305.

Woodcock, C.E., and A.H. Strahler, 1987. The factor of scale in remote sensing, Remote Sensing of Environment, 21:311-332.

Yang, H., F. van der Meer, W. Bakker, and Z.J. Tan, 1999. A backpropagation neural network for mineralogical mapping from AVIRIS data, International Journal of Remote Sensing, 20:97-110.

(Received 22 October 2002; accepted 17 January 2003; revised 18 February 2003) 